\title{
Liquid-Phase Electron Microscopy for Studying the Interaction of Two Membrane Protein Species in Overexpressing Cancer Cells
}

\author{
$\underline{\text { Diana B. Peckys }}^{1}$, Daniel Gaa $^{2}$, Florian Weinberg ${ }^{2}$, and Niels de Jonge ${ }^{2,3}$ \\ ${ }^{1}$ Department of Biophysics, Saarland University, 66421 Homburg, Germany. \\ ${ }^{2}$ INM - Leibniz Institute for New Materials, 66123 Saarbrücken, Germany. \\ ${ }^{3}$ Department of Physics, Saarland University, 66123 Saarbrücken, Germany.
}

We report on a method for simultaneous detection of single molecules of two different, but interacting membrane proteins, the epidermal growth factor receptor (EGFR) and its family member, the human epidermal growth factor receptor 2 (HER2). Both receptors are overexpressed in various types of cancer, where they trigger uncontrolled cell growth and cancer cell dissemination. To enter an activated state causing cell proliferation, the receptors have to form dimers, with themselves (homodimers) or with another receptor species (heterodimers). In EGFR-, or HER2 overexpressing cancer cells such heterodimerization is difficult to study at the single-molecule level, due to the high surface density of these receptors, reaching levels of $>1000 / \mu \mathrm{m}^{2}$ [1]. Therefore, new analytical methods are needed to better understand their interactions, for instance when evaluating anti-cancer drugs targeting EGFR or HER2.

To study the interaction of EGFR and HER2 in the plasma membrane of intact, HER2-overexpressing breast cancer cells, we developed a double-labeling protocol with two distinct types of quantum dot nanoparticles (QDs), namely QD655 and QD565. These QDs can be discerned by their size and shape, and they also have different fluorescence signatures. Both receptor species were detected in parallel with correlative, quantitative light microscopy (LM) and liquid phase scanning transmission electron microscopy (STEM) of whole cancer cells, covered with graphene sheets to keep the cells in hydrated state [2]. Due to steric hindrance, nanoparticle-labeling is generally known to leave a fraction of the target proteins unlabeled. To quantitatively characterize EGFR-HER2 heterodimers, it was thus first necessary to calculate the labeling efficiencies of each QD type for its respective target receptor species. [3]. We measured the labeling efficiencies via a newly developed method involving the determination of the cumulative distribution function (CDF) of each receptor in a cancer cell population via LM [3]. As first step, two calibration curves were created, converting fluorescence intensities values of QD655-labeled EGFR, and of QD565-labeld HER2, as measured on the cells by LM, into the underlying surface densities of the QDs, determined on the same cellular regions with LP-STEM. Fluorescent dye-conjugated binding molecules yielded CDFs at $\sim 100 \%$ labeling efficiency. Comparable CDFs were created with either QD655-labeled EGFR or QD565-labeled HER2. The differences between the median value of the EGFRor HER2 CDF derived with the fluorescent dye, and the median of the CDF with QD655 labeled EGFRor QD565 labeled HER2, revealed the labeling efficiencies for each receptor species.

The knowledge of the two QD labeling efficiencies was then used to analyze single molecule EGFR- and HER2-position information, collected from many thousands of receptors in LP-STEM images of doublelabeled cells.

The results showed that the absolute surface density of heterodimers varied between different types of functionally distinct areas of the plasma membrane. The relative fraction of EGFR assembled in homodimers was conserved, whereas the fraction of EGFR found in heterodimers markedly differed between plasma membrane regions, and was particularly pronounced in highly dynamic regions of the 
plasma membrane. These results suggest a supporting role of active EGFR in HER2 overexpressing cancer cells.

\section{References:}

[1] D Peckys et al, Molecular medicine, 25(1) 2019, p. 1.

[2] F Weinberg et al, International journal of molecular sciences, 21(23) 2020, p. 9008.

[3] D Peckys et al, Nano Letters, 20(11) 2020, p. 7948.

[4] The authors thank E. Arzt for his support through INM, the Else Kröner-Fresenius-Stiftung through the project "Investigation of the Influence of Breast Cancer Drugs on HER2 Dimerization at the Molecular Level in Individual Cells Aiming to Find Clues for Causes of Drug Resistance: HERe", and the DFG SFB1027 (project C7).

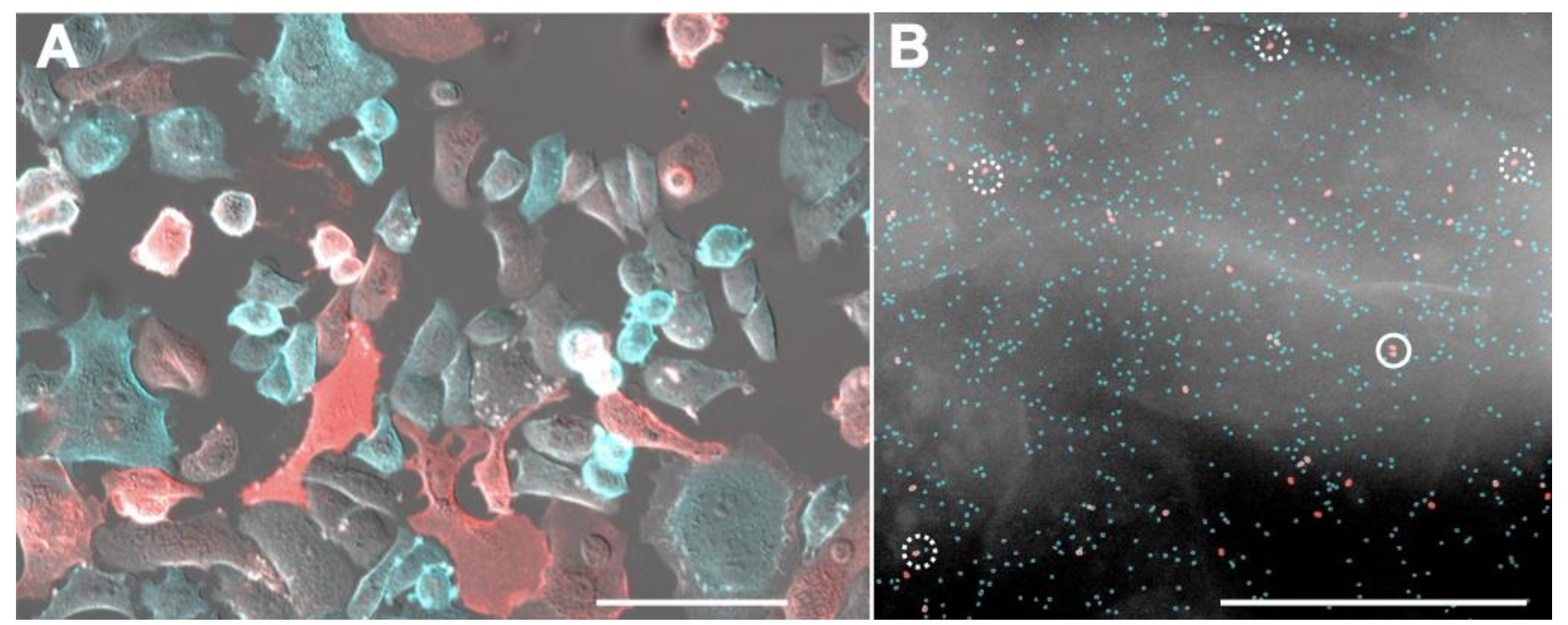

Figure 1. Correlative LM and LP-STEM of double labeled cancer cells. (A) LM image of HER2 overexpressing breast cancer cells (SKBR3), shown in an overlay of the DIC channel (exhibiting the plasma membrane surface topography), the QD655 channel, capturing fluorescence from EGFR labeled with QD655 (red), and the fluorescence intensities from the QD565 channel, originating from labeled HER2 (cyan). Note that the EGFR expression is on average more than one order of magnitude lower than the HER2 expression and the two used fluorescence images are captured with different settings. (B) A selected area of a STEM image, recorded at $120.000 \times$ magnification, and processed using automatic label detection, with QD565 outlined in cyan, and the larger QD655 in red. The surface density of HER2 is about a factor of ten higher than that of EGFR. The depicted area of the plasma membrane was a large membrane protrusion, belonging to a subset of highly dynamic regions with increased surface densities of both receptors, compared to other membrane regions. Some examples of EGFR-HER2 heterodimers are outlined with dotted circles. An exemplary EGFR homodimer is shown in the closed circle. Scale bar in A: $100 \mu \mathrm{m}$, in B: $500 \mathrm{~nm}$. 